\title{
Broadcast Communication by System Frequency Modulation
}

\author{
Douglass, Philip James; You, Shi; Heussen, Kai
}

Published in:

Proceedings of IEEE SmartGridComm 2012

Publication date:

2012

Document Version

Early version, also known as pre-print

Link back to DTU Orbit

Citation $(A P A)$ :

Douglass, P. J., You, S., \& Heussen, K. (2012). Broadcast Communication by System Frequency Modulation. In Proceedings of IEEE SmartGridComm 2012 IEEE.

\section{General rights}

Copyright and moral rights for the publications made accessible in the public portal are retained by the authors and/or other copyright owners and it is a condition of accessing publications that users recognise and abide by the legal requirements associated with these rights.

- Users may download and print one copy of any publication from the public portal for the purpose of private study or research.

- You may not further distribute the material or use it for any profit-making activity or commercial gain

- You may freely distribute the URL identifying the publication in the public portal

If you believe that this document breaches copyright please contact us providing details, and we will remove access to the work immediately and investigate your claim. 


\title{
Broadcast Communication by System Frequency Modulation
}

\author{
Philip J. Douglass, Shi You and Kai Heussen \\ Centre for Electrical Technology \\ Technical University of Denmark \\ Elektrovej-Building 325 \\ 2800 Kgs. Lyngby \\ Denmark \\ \{pjdo,sy,kh\}@elektro.dtu.dk
}

\begin{abstract}
Load controllers available today can measure AC system frequency and react to frequency deviations. A system operator can communicate to frequency sensitive loads by changing the set-points of the system's dispatchable frequency regulation resources. Explicitly signaling system state by generating off-nominal system frequency values is a novel narrowband broadcast communications channel between system operators and frequency sensitive distributed energy resources (FS-DER). The feasibility of the proposed system is evaluated on an existing island power system in Denmark. This study shows that within standard frequency quality constraints, 4 distinct symbols are feasible on the island. However, the overarching imperative of system stability prevents the symbols from having arbitrary meanings. Higher frequency values must translate into greater consumption from loads, and vice versa. Within these constraints, the proposed system would allow operators to dispatch FS-DER in a robust manner, without using an external digital control channel. By dispatching FS-DER, their well known role as a power balancing resource can be expanded to include energy balancing services.
\end{abstract}

\section{INTRODUCTION}

As non-dispatchable renewable power sources become more widely deployed, the power system as a whole must adapt to their stochastic production profile. Maintaining system stability by controlling loads to follow production is a new challenge for system operators. Loads are physically more dispersed than conventional generators, which means that existing technology for coordinating production from centralized generators is not feasible for controlling distributed energy resources (DER).

On short time scales (seconds to minutes) primary frequency regulation acts to reestablish the balance between generation and load. This made possible by high precision frequency measurements $( \pm 1 \mathrm{mHz})$ of the $\mathrm{AC}$ waveform which can be done with low-cost microelectronics. Using loads to provide frequency regulation service has been previously described in [1]-[5].

Over longer time scales (5 minutes to hours to days), loads can also be controlled to match consumption to the available energy. Whether the loads respond to a direct signal from a central controller or indirectly to a price, all systems for energy balancing rely on a digital communication system to transmit information about the desired state of the loads.

While the availability of low-cost microcontrollers allows software control of even the smallest loads, creating a communications network that reaches out to highly dispersed units is a costly endeavor. Where such a communication system has been established, maintaining the $\mathrm{N}-1$ protection criteria requires that failure of the communication system does not compromise system security, thus a fallback communication-less state must be defined.

The advantage of using system frequency to actuate highly distributed loads is that no digital communication channel is needed; the system frequency is inherently available to all buses in the synchronous system. Loads which can measure frequency for performing primary frequency control can be reprogrammed to detect different modes of operation of the power system, and in this way gain more knowledge about the system state.

Modulating AC system frequency has been used to curtail production in islanded microgrid systems with storage [6], [7]. These systems use inverters operating as voltage sources to generate a system frequency that indicates the battery state of charge. Previous work by the authors described the dispatch of frequency sensitive loads subject to time constraints [8], but the authors are unaware of previous work to analyze the feasibility of modulating frequency to convey information in an operating power system.

This paper is organized as follows. Section II analyzes the properties of system frequency when used for communicating to DER. The proposed communication system is described in detail in Section III, followed in Section IV by a case study of the island of Bornholm. Section V is a discussion and finally Section VI concludes the paper.

\section{ANALYSis of AC System FREQUENCY}

There are constraints on the range of allowable system frequencies imposed by generators and FS-DER. These 


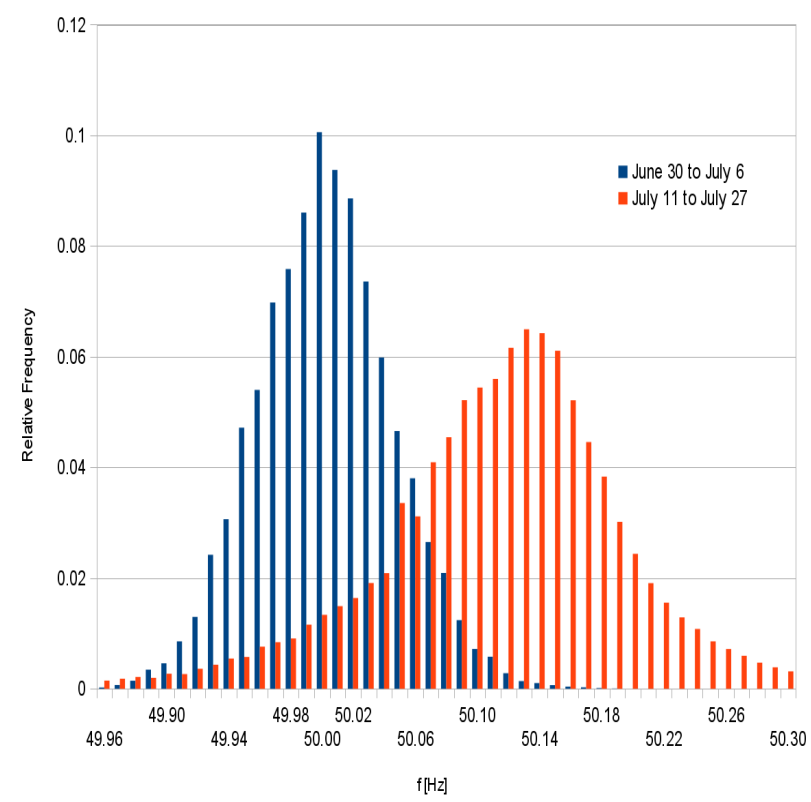

Figure 1. Relative frequency of system frequency samples a from small island power system divided into two time periods. The distinct frequency distributions were caused by two different diesel generators.

constraints will limit the number of symbols that can be encoded by modulating system frequency.

A field test of FS-DER providing primary frequency regulation is under way on the Danish islands of Bornholm (population $\approx 50,000$ ) and Christians $\varnothing$ (population $\approx 100$ ) [9]. On Christians $\varnothing$, a problem was observed with the experiment because the devices assumed the system's mean value for frequency was always the nominal frequency $(50 \mathrm{~Hz})$, like in the large interconnected Nordic power system, but on the island, the system frequency was observed at off-nominal values for extended periods of time. Fig. 1 shows the distribution of frequency data collected from Christians $\varnothing$ divided into two different time periods. It is clear from the data that the system was operated in two distinct frequency modes and this observation lead us to investigate whether these frequency mode changes could be detected by our controller and utilized to communicate information about the power system's state.

\section{A. Generators}

Large steam turbines are optimized to operate in a narrow range of frequencies and are vulnerable to frequency excursions. When electric energy is delivered from rotating machines, the swing equation dictates that system frequency will decline when the load exceeds production and frequency will rise when there is overproduction. Avoiding the upper limit on frequency is achieved by curtailing electricity production, an option that is always available. Avoiding the lower frequency limit is more costly to satisfy because it requires reserves. Grid codes in several countries allow generators operating at maximum output power to reduce output power in linear proportion to frequency when operating below a certain frequency (i.e. $49.5 \mathrm{~Hz}$ ) [10]. In a system with inadequate generation capacity, when a power imbalance pushes the system frequency below this threshold, positive feedback exacerbates the imbalance until finally the frequency declines to a level (i.e. $47 \mathrm{~Hz})$ where generator protection schemes are tripped. As a last resort to prevent total system collapse, load is shed at large granularity, causing widespread disruption.

In general, frequency regulation systems assume that changes in frequency are exogenous and that this a problem to be corrected as soon as possible, first with a droop response to stabilize the frequency and then a slower secondary control loop, such as an isochronous governor, responds to the integral of the frequency deviation to restore frequency to the set-point value. The proposed system would modify the settings of the droop to produce scheduled power at off-nominal frequencies and use the secondary control system to target off-nominal frequencies.

\section{B. Loads}

The EN 50160 standard [11] used in Europe specifies the physical characteristics of electric energy delivered to customers. Loads connected to a large interconnected power system must tolerate a frequency specified as:

$50 \mathrm{~Hz} \pm \% 1$ for $99.5 \%$ of the year

Loads in a small island power system have a wider tolerance, and must be designed for frequency constraints:

$50 \mathrm{~Hz} \pm \% 2$ for $95 \%$ of a week

Proposed grid codes from ENTSO-E [12] promote the deployment of FS-DER and relax the constraints on system frequency for large systems, allowing unlimited operation in the range $50 \mathrm{~Hz} \pm \% 2$.

\section{Frequency Distribution}

Observations of frequency measurements taken from several synchronous areas show that they conform to a Gaussian distribution, centered around the set-point value. The Gaussian distribution of frequency samples implies that with set-point frequency $f_{s}$ and standard deviation $\sigma$, 99.7\% of the time the frequency will be within $f_{s} \pm 3 \sigma$.

To satisfy the requirements of [11] for residing between $f_{\min }$ and $f_{\max }$ for $99.5 \%$ of the time, assuming a Gaussian distribution of system frequency around $f_{s}$ with standard deviation $\sigma$, the valid range of set-points satisfy:

$$
\left(f_{\min }+3 \sigma\right)<f_{s}<\left(f_{\max }-3 \sigma\right)
$$

System frequency will always be subject to external disturbances when unpredictable imbalances between generation and load occur. The response of the system to these transient imbalances is dominated by the system's inertia and time constants on the primary and secondary reserves. The UCTE grid codes which apply to Western Denmark [13], specify that after a contingency, the system 


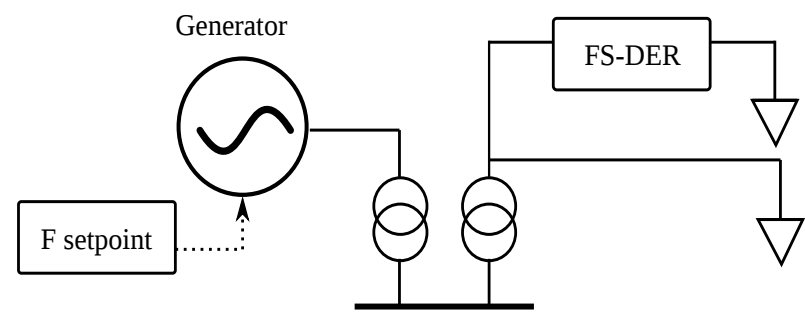

Figure 2. One-line system diagram. A lumped generator model is connected to FS-DER and conventional loads by a copper plate transmission system model. Frequency set point changes introduced to the generator are detected by the FS-DER.

frequency should be restored to $\pm 20 \mathrm{mHz}$ of the setpoint frequency within 15 minutes. This time period corresponds to the time constants for increasing production from thermal power plants. During such a contingency, the system frequency will deviate widely from the set-point. While the system is operating outside of its normal, secure operating state, FS-DER will be unable to discern the setpoint frequency. In this case, it is desirable that FS-DER contribute to restoring the system to a secure operating state.

\section{Communicating System State to Frequency SENSITIVE LOADS}

While the different frequency modes shown in Fig. 1 were unintentional, this observation shows that a system operator has latitude to reprogram frequency governors to target off-nominal system frequencies without disturbing loads. Off-nominal set-points are used today in large interconnected systems in response to clock drift. In the Nordic power system, time correction is done on occasion by human intervention [14]. It is conceivable that a synchronous area would take active use of this degree of freedom to vary frequency set-points depending on the system state (i.e. based the predicted availability of stochastic energy sources).

The control of distributed energy resources is typically classified as either direct or indirect [15]. Direct control corresponds to the architecture of existing utility SCADA systems where commands are issued from a centralized controller and remote terminals respond to the commands in a deterministic manner. Frequency controlled reserves are not usually considered as directly controlled because the control signal (system frequency) is not actively generated, but rather results from the intrinsic behavior of the power system. A system operator with knowledge of FS-DER configuration parameters can directly control them by using existing generator governors to change the setpoint frequency to reflect the system state as shown in Fig. 2.

\section{A. Generator Model}

Previous work on FS-DER has focused on its role to supplement the generators performing primary frequency

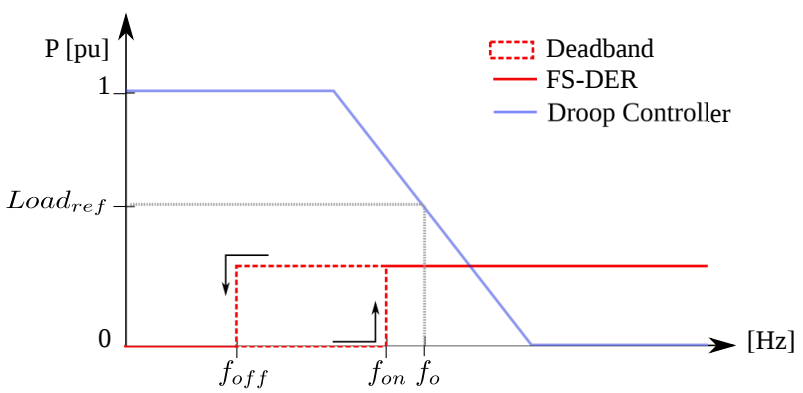

Figure 3. Deployment of FS-DER as frequency controlled reserve. Power output of dispatchable generation and power consumption of FS-DER with two states (OFF/ON) is shown as a function of system frequency. The generator delivers scheduled power $\left(\operatorname{Load}_{r e f}\right)$ at the nominal frequency $\left(f_{o}\right)$. The load is disconnected when the generator's capacity for up-regulation is exhausted.

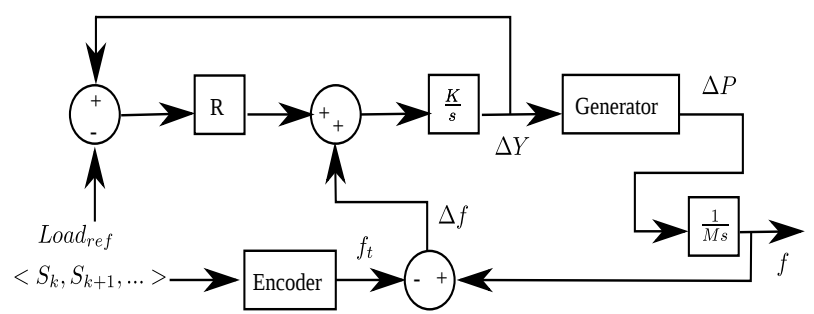

Figure 4. Simplified block diagram of a droop controller used as a transmitter, adapted from [16]. A series of symbols $\left(S_{k}, S_{k+1}, \ldots\right)$ is encoded to frequency setpoint values $\left(f_{t}\right)$ which are then given to the standard droop controller. The system's inertia $(M)$ determines how quickly a power imbalance $(\Delta P)$ changes system frequency $(f)$.

regulation [1]-[4]. Dispatchable generators perform primary frequency control with a droop controller which produces power proportional to the system frequency, with lower frequencies resulting in higher power output. The relationship between the system's primary droop response and FS-DER is shown in Fig. 3. Fig. 4 shows a block diagram of a droop controller augmented with a stream of inputs from the system operator which are encoded into frequency setpoints.

\section{B. Load Model}

The Demand as Frequency Controlled Reserve algorithm for discrete relay-based devices described in [3] has a frequency threshold $\left(f_{o f f}\right)$ below which the load is disconnected. Loads are reconnected when system frequency is above a second, higher threshold frequency $\left(f_{o n}\right)$, creating a simple deadband. This concept can be generalized to more complex DER which have more than two states of operation. For FS-DER to identify a system frequency mode $x$, the sampled system frequency $\left(f^{\prime}\right)$ must be between the lower frequency threshold $\left(f_{x-}\right)$ and the upper frequency threshold $\left(f_{x+}\right)$. The controller will transition to state $x$ when:

$$
f_{x-} \leq f^{\prime} \leq f_{x+}
$$

The thresholds would have to be chosen so that they were separated by enough distance to avoid spurious 


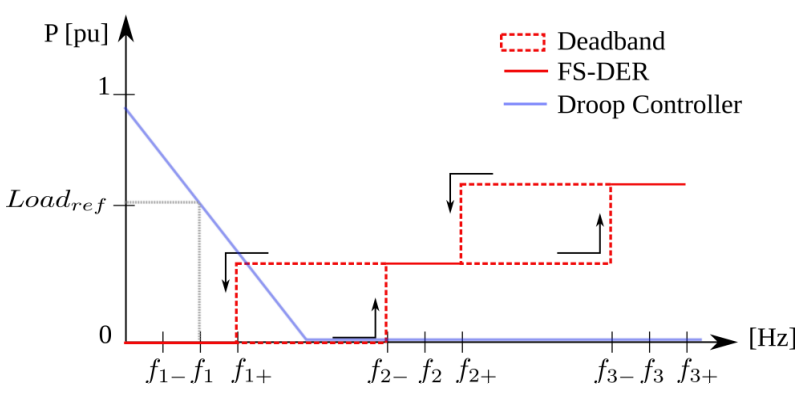

(a)

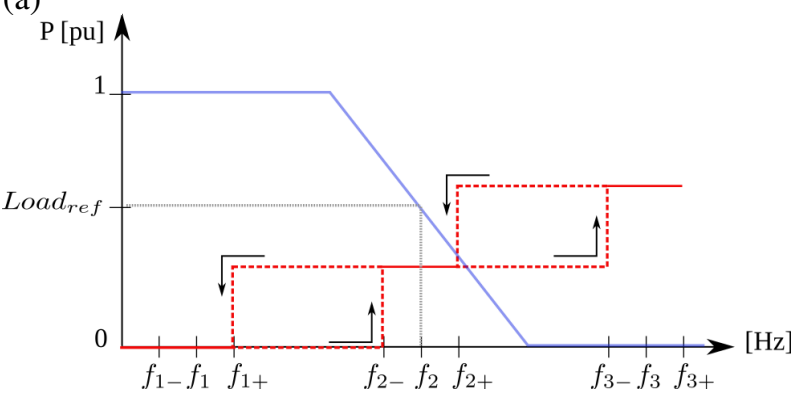

(b)

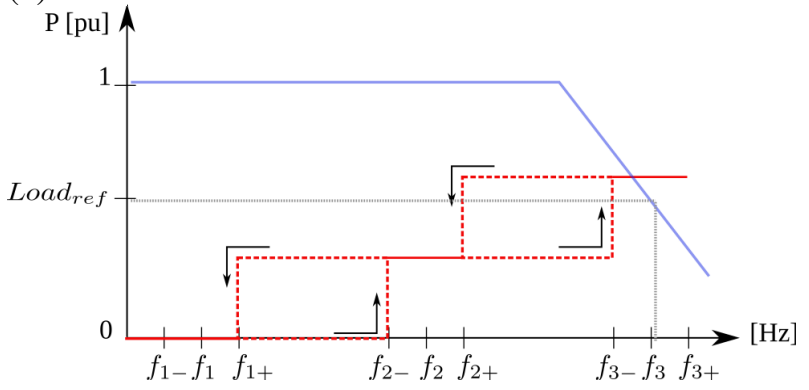

(c)

Figure 5. Power production from dispatchable generation with droop controller and consumption from FS-DER with three states. (a) Droop controller frequency setpoint at $f_{1}$. (b) Droop controller frequency setpoint at $f_{2}$. (c) Droop controller frequency setpoint at $f_{3}$.

unintended state changes. Fig. 5 shows how different frequency setpoints of a droop controlled generator can force FS-DER into one of 3 states.

To successfully communicate to DER, the system frequency should minimize the time it operates in the deadband, between two states. While the frequency is in the deadband, the probability of residing in an unintended state are higher because the FS-DER state is determined by hysteresis rather than the current frequency. A wide deadband is needed to avoid spurious changes to FS-DER state, but this forces frequency set-points to be farther from each other, reducing the number of symbols possible in a given bandwidth.

To narrow the deadband while avoiding spurious state changes, the DER can pass the frequency samples through a low pass filter before comparing the value to preset thresholds. In this way the variance of the Gaussian distribution is reduced. A similar algorithm was described in [17], where different cutoff frequencies existed for short-term frequency deviations (i.e. 1s) and longer-term

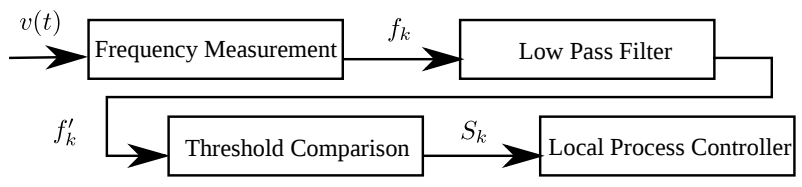

Figure 6. Block diagram of FS-DER load controller. In time period $k$, the frequency $f_{k}$ of the incoming voltage waveform $v(t)$ is filtered and then compared to pre-set threshold values, resulting in state $S_{k}$ which is given as input to the controller of the energy using process.

deviations (i.e. $5 \mathrm{~min}$.). This algorithm is well suited for the proposed scheme because a small but consistent deviation from nominal frequency on a long time scale will force FS-DER into a known state. A block diagram of the full receiver is shown in Fig. 6.

\section{Determining Frequency Threshold Values}

Both the transmitter and receiver need to agree a priori on frequency setpoint and threshold values. To find appropriate frequency thresholds for the system states, the assumption of a Gaussian distribution of frequency measurements is used. If thresholds are chosen to be $f_{s} \pm 5 \sigma$, the frequency value will be within this range $99.9999 \%$ of the time, implying that it will be outside this range 50 milliseconds per day. The frequency will be within $f_{s} \pm \sigma$ for $68 \%$ of the time. When the system operator wishes to communicate that the system is in state $S_{x}$, the encoder will set the generator's frequency set-point to $f_{x}$.

The distance from a given frequency set-point $\left(f_{x}\right)$ to the next adjacent set-point $\left(f_{x+1}\right)$ must be large enough to make spurious changes during normal operation rare. This is satisfied by ensuring

$$
f_{x}+5 \sigma \leq f_{(x+1)-}
$$

Fig. 7 shows the placement of set-points and state change thresholds for a system with 3 symbols.

When a state change is desired, the system frequency must be forced across the threshold values for long enough for the filtered value to cross the threshold. Filtering measurements over a longer time period reduces the maximum rate of change of symbols.

\section{CAse Study: Bornholm}

Bornholm, an island in the Baltic Sea, is normally connected to Sweden by means of a $60 \mathrm{kV}$ AC cable. In April 2012, Bornholm operated as an electrical island, disconnected from Sweden. During this time, over a period of 4 days, high resolution frequency measurements were taken from a $60 \mathrm{kV}$ substation. These frequency measurements were analyzed at $1 \mathrm{~s}$ intervals to characterize the system frequency when operating as an electrical island.

The frequency measurements conform well to a normal distribution, with a average of $50.002 \mathrm{~Hz}$ and $\sigma=41$ $\mathrm{mHz}$ over the time period. The maximum frequency measurement was $50.200 \mathrm{~Hz}=f_{o}+4.87 \sigma$, the minimum 


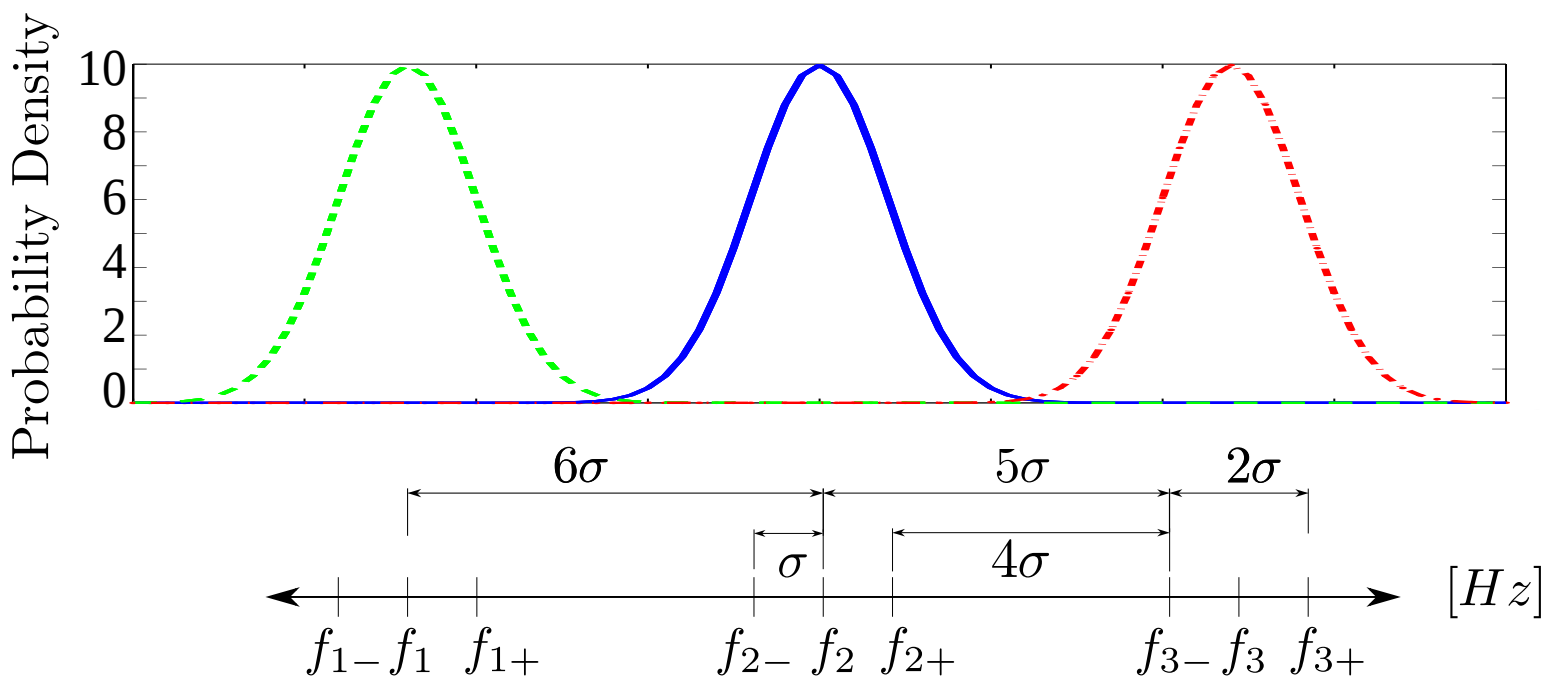

Figure 7. Top: Probability density of frequency samples for three system frequency setpoints: $f_{1}, f_{2}, f_{3}$. Below: The placement of set-points and the threshold values.

Table I

SUGGESTED PARAMETER VALUES FOR FREQUENCY SET-POINTS ON BORNHOLM WITH 4 SYMBOLS

\begin{tabular}{|l|l|l|}
\hline Parameter & Relative Value & Value \\
\hline$f_{o}$ & & $50 \mathrm{~Hz}$ \\
$\sigma$ & & $40 \mathrm{mHz}$ \\
\hline$f_{1}$ & $f_{2}-6 \sigma\left(f_{\min }\right)$ & $49.64 \mathrm{~Hz}(49.62 \mathrm{~Hz})$ \\
$f_{1+}$ & $f_{1}+\sigma$ & $49.68 \mathrm{~Hz}$ \\
\hline$f_{2-}$ & $f_{2}-\sigma$ & $49.84 \mathrm{~Hz}$ \\
$f_{2}$ & $f_{o}-3 \sigma$ & $49.88 \mathrm{~Hz}$ \\
$f_{2+}$ & $f_{2}+\sigma$ & $49.92 \mathrm{~Hz}$ \\
\hline$f_{3-}$ & $f_{3}-\sigma$ & $50.08 \mathrm{~Hz}$ \\
$f_{3}$ & $f_{o}+3 \sigma$ & $50.12 \mathrm{~Hz}$ \\
$f_{3+}$ & $f_{3}+\sigma$ & $50.16 \mathrm{~Hz}$ \\
\hline$f_{4-}$ & $f_{4}-\sigma$ & $50.32 \mathrm{~Hz}$ \\
$f_{4}$ & $f_{3}+6 \sigma\left(f_{\max }\right)$ & $50.34 \mathrm{~Hz}(50.38 \mathrm{~Hz})$ \\
\hline
\end{tabular}

measurement was $49.806 \mathrm{~Hz}=f_{o}-4.73 \sigma$. Taking a moving average of samples over 1 minute marginally reduces the standard deviation to $\sigma=38 \mathrm{mHz}$ (max: $50.163 \mathrm{~Hz}=f_{o}+4.29 \sigma$, min: $\left.49.846 \mathrm{~Hz}=f_{o}-4.05 \sigma\right)$, averaging over 10 minutes results in $\sigma=35 \mathrm{mHz}$ (max: $50.135 \mathrm{~Hz}=f_{o}+3.86 \sigma$, min: $\left.49.87 \mathrm{~Hz}=f_{o}-3.23 \sigma\right)$. Averaging frequency samples smooths the extrema of the frequency distribution, but the reduction in standard deviation is not larger because the system exhibits offnominal frequencies for long durations.

Assuming that Bornholm wished to live up to the requirements of an interconnected power system in [11], and with $\sigma=40 \mathrm{mHz}$, it follows from eq. (1) that the range of possible system frequency set-points is between $f_{\min }=49.62 \mathrm{~Hz}, f_{\max }=50.38 \mathrm{~Hz}$. The available bandwidth is:

$$
50.38 \mathrm{~Hz}-49.62 \mathrm{~Hz}=0.76 \mathrm{~Hz}=18.5 \sigma
$$

Suggested values for frequency set-points and thresholds for 4 symbols are shown in table I.

If the system operator on Bornholm instead aimed for the more lenient requirements for a small power system, the available bandwidth would be:

$$
50.88 \mathrm{~Hz}-49.12 \mathrm{~Hz}=1.76 \mathrm{~Hz}=44 \sigma
$$

In this case, a separation distance between symbols of $6 \sigma$ would allow for 8 distinct states to be communicated from an operator to FS-DER.

\section{Discussion}

Parameter values for the proposed AC frequency modulation system will vary greatly between synchronous systems. This operating method is most relevant for small synchronous systems because they already have a relatively wide range for their system frequency, and their generally smaller capacity margins motivate the use of energy management systems. Large synchronous systems have less available bandwidth but this is mitigated by larger inertia and smaller frequency variance, so the distance between symbols can be smaller.

The number of symbols that can be encoded with system frequency modulation is a trade-off between large offnominal frequencies that reduce margins for frequency stability, and smaller spacing between set-point frequencies with an increased probability of communication errors to FS-DER. Low pass filtering of frequency samples can reduce the spacing between set-points without compromising error-rate, but results in slower transition time from one state to another. The maximum rate of change of frequency offsets, and hence the baud rate, will be constrained by the system's inertia and the dynamic properties of the rotating machines.

Analysis of a system's frequency stability will have to account for operation at off-nominal frequencies, which may result in the need for more frequency regulation resources. The operator's load control performance would 
depend on how closely the state received by FS-DER conformed to state intended by the operator.

The difference between frequency setpoint values would have to be larger than the typical variation of frequency (i.e. $>5 \sigma$ ) but if disturbances pushed the system frequency into a neighboring frequency band, the action of the FSDER will act to restore the frequency to the targeted value. This means that the symbols can not have arbitrary meaning, higher frequencies must translate into higher consumption (and less production) in aggregate.

The frequency regulation function of FS-DER can coexist with the proposed communication scheme if the FSDER contained different cut-off thresholds: one threshold for fast response to serious disturbances, and another threshold for detecting system state changes using the lowpass filtered frequency samples.

Ideally, frequency set points would be adjusted equally above and below nominal to prevent clock drift, but if clocks are the only application which relies on this time service, it can be sacrificed because there are plenty of alternatives for finding the time of day.

Many loads are indifferent to variations in system frequency, but off-nominal frequency may lead to higher losses in machines optimized for a particular frequency (such as generators). This concept could have a negative effect on machine efficiency, however it is targeted towards applications where energy production capacity is the dominating constraint.

\section{CONCLUSION}

The paper has described a new operating concept which broadcasts discrete system state information to frequency sensitive distributed energy resources (FS-DER). System state is communicated to FS-DER by adjusting the generators' frequency controller to target off-nominal frequencies. The number of distinct states that a load can detect is a function of the bandwidth available and the standard deviation of frequency measurements. The standard deviation in turn, can be reduced by a low pass filter on raw frequency samples. The feasibility of this concept was demonstrated by analyzing data collected from an operating island power system. The analysis shows that FS-DER loads can be dispatched into 4 discrete states while conforming to standard frequency constraints.

Future work will seek to characterize the rate of change of frequency setpoints for different types of synchronous systems.

\section{REFERENCES}

[1] J. Short, D. Infield, and L. Freris, "Stabilization of grid frequency through dynamic demand control," Power Systems, IEEE Transactions on, vol. 22, no. 3, pp. $1284-1293$, aug. 2007.

[2] P. Nyeng, J. Østergaard, M. Togeby, and J. Hethey, "Design and implementation of frequency-responsive thermostat control," in Universities Power Engineering Conference (UPEC), 2010 45th International, 31 2010-sept. 3 2010, pp. 1 -6.

[3] Zhao Xu, J. Østergaard, and M. Togeby, "Demand as frequency controlled reserve," Power Systems, IEEE Transactions on, vol. 26, no. 3 , pp. $1062-1071$, aug. 2011.
[4] D. P. Chassin, M. K. Donnelly, and J. E. Dagle, "Electrical power distribution control methods, electrical energy demand monitoring methods, and power management devices," US Patent US 8073573,12 06, 2011. [Online]. Available: http://availabletechnologies.pnnl.gov/media/61_126201224640.pdf

[5] D. Trudnowski, M. Donnelly, and E. Lightner, "Power-system frequency and stability control using decentralized intelligent loads," in Transmission and Distribution Conference and Exhibition, 2005/2006 IEEE PES, may 2006, pp. 1453 -1459.

[6] P.-O. Moix, "A Minigrid of Individual Solar Home Systems," in 6th European Conference of PV-Hybrids and Mini-Grids, 2012.

[7] Steca Elektronik GmbH, "Steca RCC-02 Operating Instructions," Online, accessed February 9, 2012. [Online]. Available: http://www.stecasolar.com/index.php?Steca_Xtender_Zubehoer_en

[8] P. Douglass and S. You, "Direct Load Control by AC frequency Modulation," in 6th European Conference of PV-Hybrids and MiniGrids, 2012.

[9] P. Douglass, R. Garcia-Valle, P. Nyeng, J. Østergaard, and M. Togeby, "Demand as Frequency Controlled Reserve: Implementation and practical demonstration," Innovative Smart Grid Technologies Europe, 2011.

[10] I. Marchando and I. Arian, "Grid Codes Comparison," Master's thesis, Chalmers University of Technology, 2006.

[11] Standard, "EN 50160: Voltage characteristics of electricity supplied by public electricity networks," 2010.

[12] ENTSO-E, "Draft Demand Connection Codes," 27 Jul. 2012.

[13] UCTE, "Operations Handbook Appendix 1: Load Frequency Control," 16 Jun. 2004.

[14] Nordel, "System operation agreement," Online https://www.entsoe.eu/, 13 Jun. 2006, accessed April 27, 2012. [Online]. Available: https://www.entsoe.eu/

[15] D. Callaway and I. Hiskens, "Achieving controllability of electric loads," Proceedings of the IEEE, vol. 99, no. 1, pp. $184-199$, jan. 2011.

[16] P. Kundur, Power System Stability and Control. McGraw Hill, 1994.

[17] A. Molina-Garcianda, F. Bouffard, and D. Kirschen, "Decentralized demand-side contribution to primary frequency control," Power Systems, IEEE Transactions on, vol. 26, no. 1, pp. $411-419$, feb. 2011. 